\title{
A Method for Evaluating the Relationship among Four Perspectives of the Balanced Scorecard
}

\author{
Yumiko Taguchi ${ }^{1}$, Takumi Kaneko ${ }^{2}$, and Tsutomu Tabe ${ }^{2}$ \\ ${ }^{1}$ Department of Business Administration and Communication, Shohoku College, \\ 428 Nurumizu, Atsugi, Kanagawa 243-8501 Japan \\ ${ }^{2}$ Department of Industrial and Systems Engineering, College of Science and \\ Engineering, Aoyama Gakuin University, 5-10-1 Fuchinobe, Sagamihara, \\ Kanagawa 229-8558 Japan \\ taguchi@shohoku.ac.jp, c5605046@cc.aoyama.ac.jp, \\ tabe@ise.aoyama.ac.jp
}

\begin{abstract}
The purpose of this study is to clarify a method for quantitatively evaluating the relationship among the four perspectives of the BSC. In order to establish the purpose of this study, we (1) clarify the methodology to quantitatively understand the vision and relationship among the four perspectives, (2) analyze the KPI data of a corporation model which is similar to practical business with the evaluation method shown in this study and (3) verify the practical effectiveness of the method based on the obtained results.
\end{abstract}

Keywords: Balanced scorecard, four perspectives, balance, high-order factor analysis, covariance structure analysis.

\section{Introduction}

Changes in various perspectives of business management are strongly requested due to recent economic globalization and increased competition. The balanced Scorecard (BSC) is gathering attentions as one of these management systems [1]. In the BSC, specific targets (strategic objectives) required for executing strategies (company strategy, business strategy, etc) to accomplish the vision of a company constructed from the status of the future industry and development of the company are categorized into four perspectives: "Financial," "Customer," "Internal Business Processes" and "Learning and Growth." The strategic objectives categorized into these four perspectives further evolve into each Critical Success Factor (CSF), which accomplishes the four perspectives respectively, and the Key Performance Indicator (KPI) to quantitatively evaluate CSF.

By the way, David P. Norton, who is one of the developers of the BSC uses the term "Unbalanced Scorecard", meaning "bad operation status of the BSC." It is a state of the BSC in which the balance between the financial perspective and the other three perspectives is completely lost. There are many cases in which the financial perspective is significantly stronger than the other perspectives and benefits of the BSC, and thus "balance" is not received at all. Therefore, an organization should be evaluated and 
improved by keeping balance among the four perspectives while focusing on vision and strategy when executing the BSC.

Previous studies reported methods for quantitatively understanding the causal relationship among strategic objectives and the pervasion level of strategy [2][3]. However, no study has reported a method for evaluating and improving the balance among the four perspectives.

Hence, the purpose of this study is to show a method for evaluating the relationship among the four perspectives of the BSC. In order to establish the purpose of this study, we (1) present the methodology to quantitatively understand the vision and relationship among the four perspectives, (2) analyze the KPI data of a corporation model which is similar to practical business with the evaluation method proposed in this study and (3) verify the practical effectiveness of the method based on the obtained results.

\section{A Method for Quantitatively Understanding the Relationship among the Four Perspectives}

At first, a model expressing the status of the balance is constructed. Then, an analysis method applied to the model is discussed. Finally, we propose a method for evaluating the balance through the analysis method.

\subsection{A Model Expressing the Status of the Balance of the BSC}

Balances in the BSC can be categorized into the balance between short and long-range objectives, the balance among the past, present and future, the balance between financial and non-financial perspectives, and the balance between external and internal perspectives.

The balance has no fixed criterion (i.e., distribution by percentage). It is important to build a relationship as expected and predicted by a business manger or the person who prepared the BSC.

Therefore, we evaluate the relationship among the four perspectives in this study. These four perspectives were created from vision and strategy. It is difficult to make an evaluation based only their relationship. Thus, we "evaluate the relationship among the four perspectives" by "quantitatively indicating the relationship among the four perspectives."

We construct a model for the relationship between the vision, strategy, four perspectives and strategic objective, and the KPI. This model is called an evolutionary model of the BSC.

We should note that the KPI (key performance indicator) is quantitative data, while the strategic objective, four perspectives and vision are qualitative data (i.e., concepts) in this study. The method used to analyze this model will be specified while paying attention to this respect.

\subsection{Discussion of the Analytical Method}

We should consider the following four issues concerning the method for analyzing the evolutionary model of the BSC: We can (1) freely construct the model to be analyzed. 
(2) Analyze the model including concepts that cannot be directly measured. (3) Distinguish the relationship among variables. (4) Analyze the relationship among qualitative data.

We use high-order factor analysis in order to consider these issues in this study. High-order factor analysis is "a covariance structure analysis and a model assuming higher concepts exist behind the factors supposed to be at the back of quantitative data." We can construct a model specific to the data according to the data format by the covariance structure analysis. A variable that can be measured is called an "observed variable." A factor that cannot be directly observed is called a "constructive concept." We can analyze the causal relationship by including constructive concepts using this method.

In other words, by using high-order factor analysis, we can quantitatively evaluate the relationship among factors that cannot be directly explained by observed variables and analyze models by utilizing the method to evolve the BSC without modification. More specifically, when the evolutionary model of the BSC is applied to high-order factor analysis, as shown in Fig. 1, we can quantitatively evaluate the relationship between the vision and the four perspectives from the path coefficient between the second and third-order factors. Because the model is applied by assuming the KPI data to be observed variables, the strategic objective to be the first-order factor, the four perspectives to be the second-order factors, and the vision to be the third-order factor.

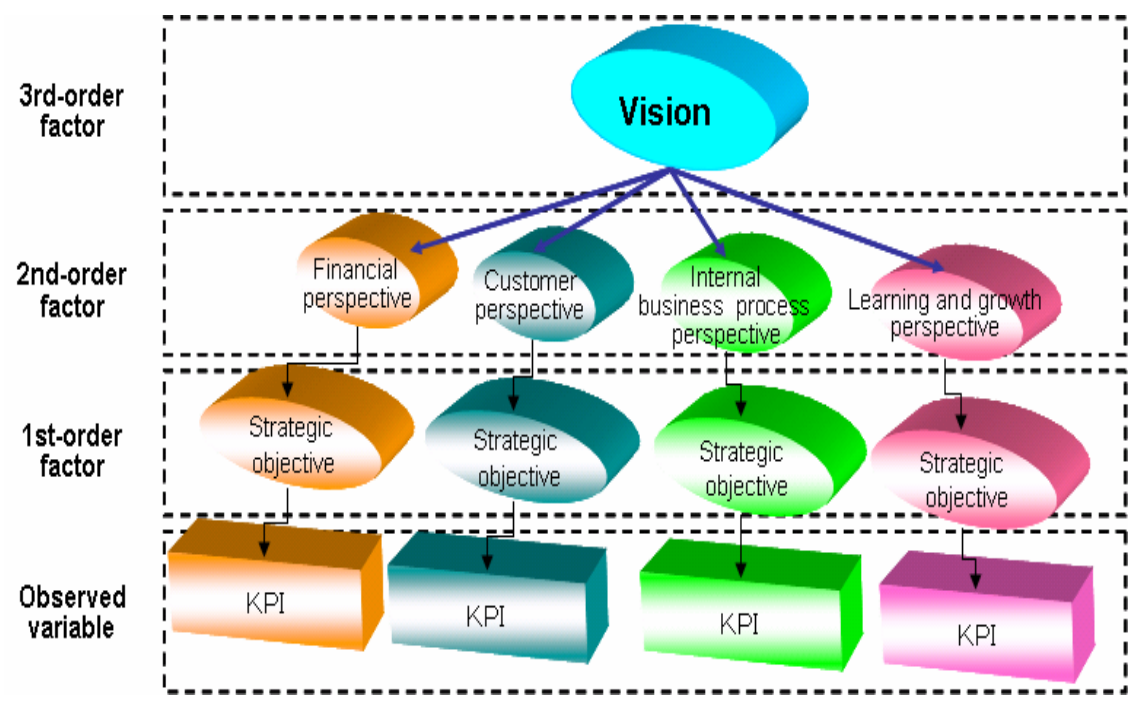

Fig. 1. Setting evolutionary model and high-order factors

\subsection{A Method for Evaluating Balance}

In this study, balance of each perspective is a ratio obtained from the following equation (1) based on the path coefficient of the vision and four perspectives. 


$$
X_{i}=\frac{x_{i}}{x_{1}+x_{2}+x_{3}+x_{4}} \times 100
$$

where

$X_{i}$ :balance of perspective $i(\%)$

$x_{i}$ :Path coefficient of vision and perspective $i$

$i=1$ : Financial perspective

$i=2$ : Customer perspective

$i=3$ : Internal business process perspective

$i=4$ : Learning and growth perspective

We used high-order factor analysis in the evolutionary model of the BSC. As a result, we could not always construct the relationship between vision and the four perspectives as expected.

If no relationship was constructed as expected, approaches after that were very important. As shown in Fig. 2, we prepare a chart to interpret the analysis results and trace the relationship between the four perspectives and strategic objectives, and the strategic objectives and KPIs in the chart to identify causes of the imbalance.

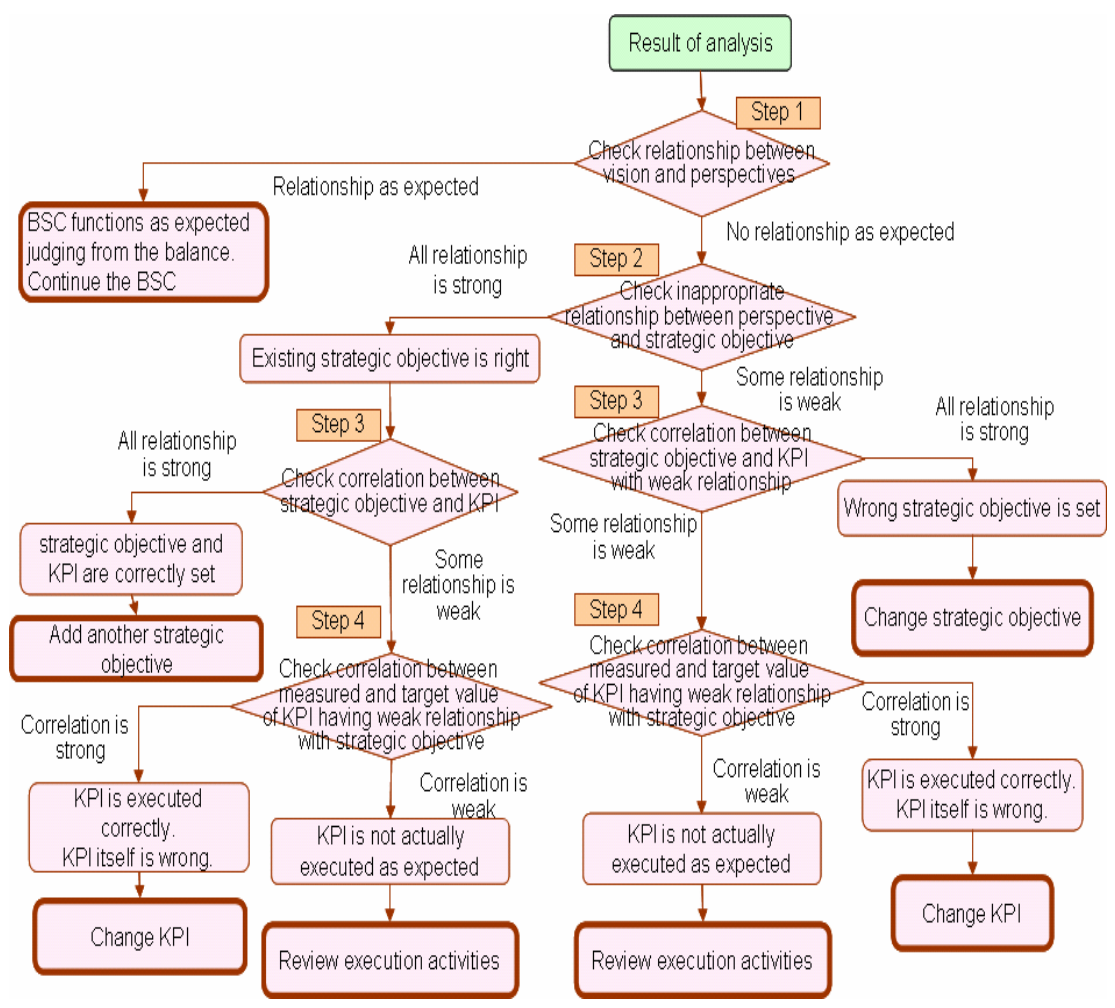

Fig. 2. Chart to interpret results of the analysis 
The interpretation chart consists of four steps: (1) checking the relationship between vision and perspectives. (2) checking the inappropriate relationship between perspectives and strategic objectives. (3) checking the relationship between strategic objectives and KPIs. (4) checking the correlation between the actual value and target value of KPIs which have a weak relationship with strategic objectives. The strength of the relationship of (1) through (3) shown above is determined by the path coefficient obtained from the high-order factor analysis.

\section{Discussion of the Effectiveness}

We prepare the BSC of a corporation model simulating actual business conditions in order to discuss the effectiveness of the evaluation method shown above. We verify the following two issues based on the KPI data of the BSC. First, we construct a "balanced model" and "unbalanced model" and perform a high-order factor analysis. We determine whether there is an expected relationship between the vision and the four perspectives.

Second, we construct "a model in which inappropriate KPIs are set" by design. We verify whether the result affects the relationship between the vision and perspectives. We further determine whether we can extract "inappropriate KPI" which are the cause.

\subsection{Corporate Model}

We use data consisting of actual data provided by a corporation, as well as virtual data. We asked a businessperson to check the virtual data prepared in this study. The businessperson considered the virtual data to be useful.

The corporate model $\mathrm{X}$ is a manufacturer of resin. They have a vision "to provide good products and solutions aiming at business development with customers." The prepared BSC consists of a company-wide BSC, a BSC of the production department, and a BSC of the sales department.

\subsection{Verification 1}

As shown in Fig.3, we applied high-order analysis to the evolutionary model of the company-wide BSC for the "balanced model." Meanwhile, we applied high-order factor analysis to the evolutionary model of the BSC of the production department for the "imbalanced model."

It is estimated that the four perspectives are well-balanced in the evolutionary model of the company-wide BSC. On the contrary, it is also estimated that the "internal business process perspective" and "learning and growth perspective" account for a larger ratio than the "financial" and "customer" in the evolutionary model of the BSC of the production department. We applied high-order factor analysis on these two models by using AMOS, which is covariance structure analysis software. We obtained the following results:

In the evolutionary model of the company-wide BSC, financial, customer, internal business process, learning and growth had an effect of $25 \%, 25 \%, 26 \%$ and $24 \%$, respectively, showing an almost equal ratio. Meanwhile, financial, customer, internal business process, learning and growth accounted for 19\%, 24\%, 28\%, 29\%, 
respectively, in the evolutionary model of the BSC in the production department. The "internal business process" and "learning and growth" perspectives had larger ratio in their effect. As shown above, the high-order factor analysis can show the relationship among the four perspectives in a quantitative manner.

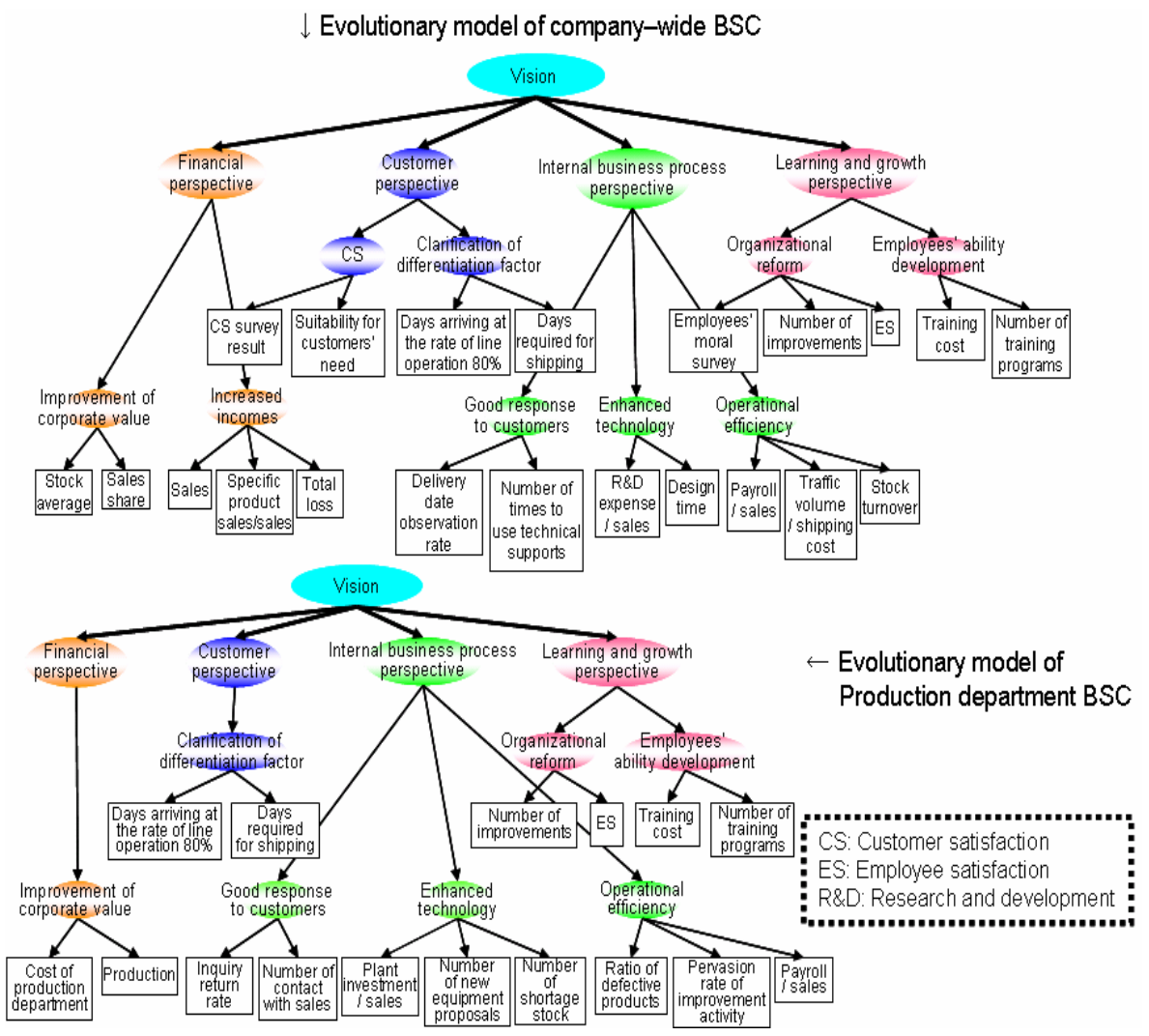

Fig. 3. Evolutionary BSC models company-wide and of the production department

\subsection{Verification 2}

As shown in Fig. 4, we set the "training cost", supposed to be a KPI of learning and growth perspective, in the KPI for the strategic objective of customer perspective in the evolutionary model of the BSC of the sales department. We confirm whether the setting is reflected in the results or not. Normally, it is estimated that the "financial" and "customer" perspectives account for a larger ratio than the "internal business process" and "learning and growth" perspectives in the evolutionary model of the BSC of the sales department.

We performed a high-order factor analysis as in verification 1 shown above. The following results were obtained: The four perspectives account for 50\%, 8\%, 36\% and $6 \%$ respectively. The four perspectives were imbalanced. We tried to identify the cause using the "Chart to interpret analysis results." We obtained the results shown in Fig. 5. 


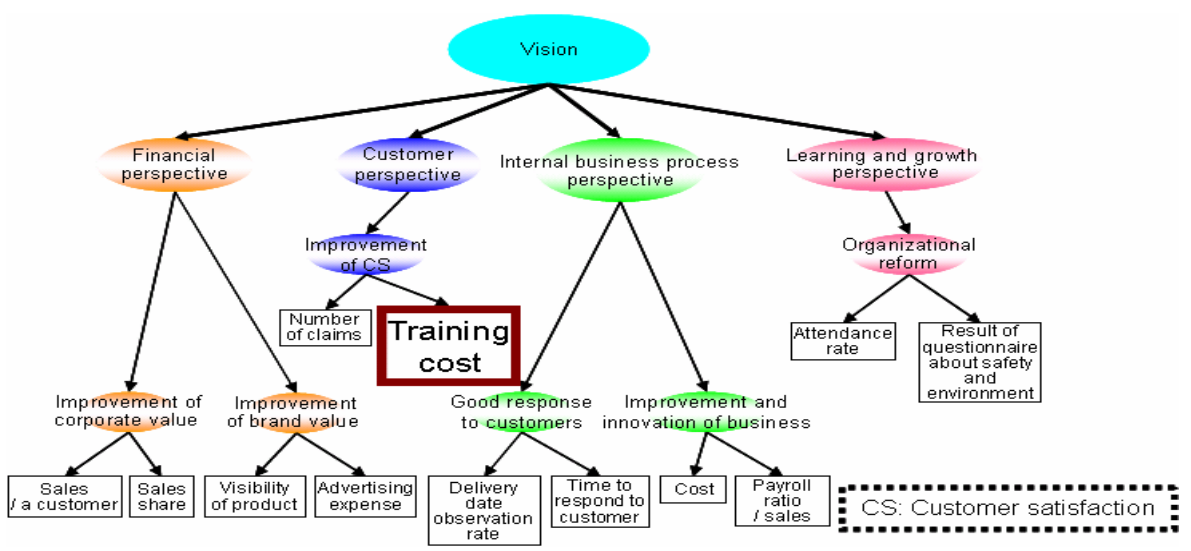

Fig. 4. BSC evolutionary model of the sales department

In step 2 of the interpretation chart, we check the relationship between inappropriate customer perspective and the improvement of CS, which is a strategic objective. The path coefficient between the two factors is -0.99 , showing a strong relationship. Therefore, the improvement of CS is felt to be appropriately set for customer perspective.

Then, in step 3, we check the relationship between improvement of CS, and the number of complaints and training cost which are KPIs of improvement of CS. The path coefficient between the improvement of CS and the number of complaints is -0.99 (strong relationship). Meanwhile, the path coefficient between improvement of CS and training cost is 0.34 (weak relationship). Consequently, in step 4, we check whether or

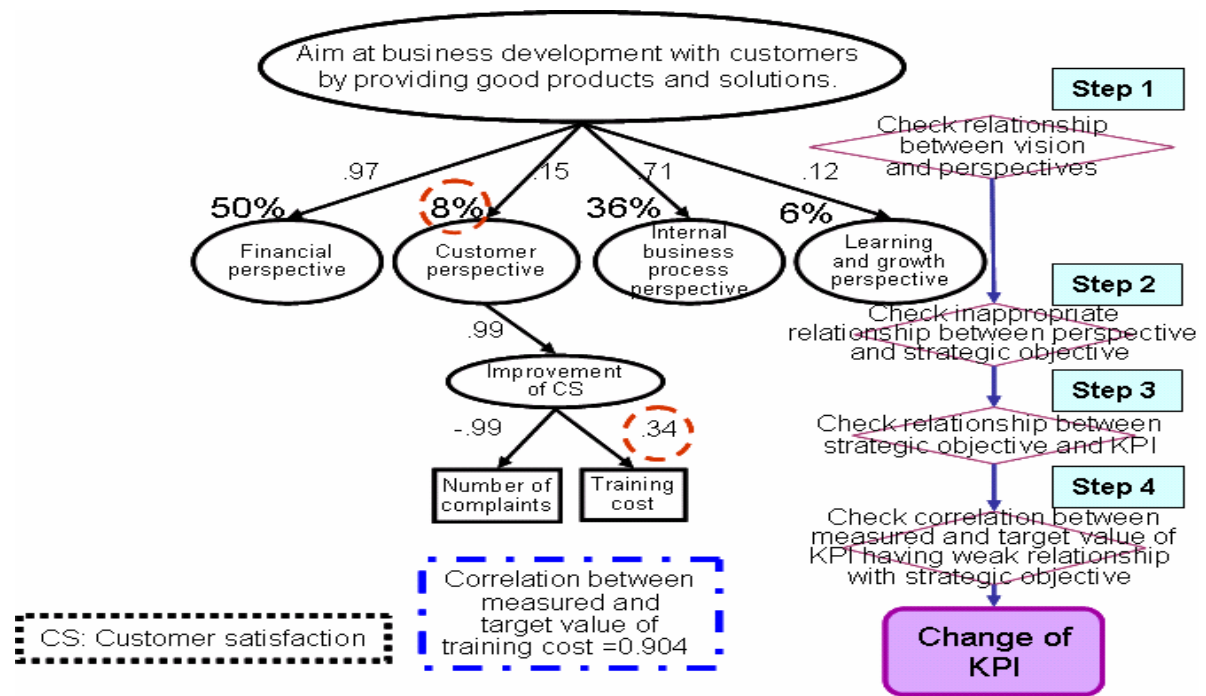

Fig. 5. Analysis results of BSC in the sales department 
not the setting of the training cost itself was appropriate by using the correlation between the measured value and the target value of the training cost. As a result, the value is 0.9 , showing a strong correlation. Therefore, we understand that the imbalanced customer perspective in the BSC of the sales department was caused by an inappropriate setting of KPI (training cost).

\section{Conclusion}

We demonstrated the usability of an evaluation method using high-order factor analysis as a methodology to quantitatively understand and evaluate whether or not a balanced BSC is constructed in the relationship of the four perspectives in this study. In the future, we should further investigate how to apply data including the relationship between the target value and measured value as the handling of observed variable data, perform analyses and extend our knowledge.

\section{References}

1. Kaplan, R.S., Norton, D.P.: The balanced scorecard-measures that drive performance. Harvard Business Review 70(1), 71-79 (1992)

2. Kaplan, R.S., Norton, D.P.: Using the Balanced Scorecard as a Strategic Management System. Harvard Business Review 85(7-8), 150-161 (2007)

3. Taguchi, Y., Ota, M., Tabe, T.: A method for quantitatively appraising strategy penetration in BSC. In: 2nd International Conference on Applied Human Factors and Ergonomics, CD-ROM (2008) 